Bolm Inst. oceanogr., S Paulo, 32(1):63-69, 1983

\title{
BIOLOGIA REPRODUTIVA DE MICROPOGONIAS FURNIERI (DESMAREST, 1823) (TELEOSTEI, SCIAENIDAE), 1. FATOR DE CONDIÇÃO COMO INDICADOR DO PERİODO DE DESOVA
}

\author{
Victōria Judith ISAAC-NAHUM ${ }^{1 *}$ \& Anna Emília Amato de Morais VAZZOLER ${ }^{2 * *}$. \\ 1 SUDEPE - Superintendência do Desenvolvimento da Pesca (SP) \\ 2 Instituto Oceanográfico da Universidade de São Paulo
}

\section{Synopsis}

The monthly analysis of maturity stage frequency of adult females caught monthly at the Ubatuba region, SP, from February 1979 to January 1980, suggests that M. furnieri spows three times a year: Autumn (April-June), Winter (August-September) and Spring-Sumer (November-February). Condition factor fluctuations estimated with andwithout gonads ( $K$ and $K^{\prime}$ ) during a reproductive cycle reinforces hypothesis. $K$ and $K^{\prime}$ maximum values relate precisely with higher frequency periods of a females at advanced stages of maturity (C). On the other hand, $K$ and $K^{\prime}$ minimum values are concomitant with higher frequency periods of spent females (D). Although $K$ and $K^{\prime}$ have a similar evolution, there are some periods in which $\Delta K\left(K-K^{\prime}\right)$ appears higher: May, August-September and November. These periods are also related to the maximum frequency of females in stage C. As $\Delta K$ estimes the "gonad condition", establishing the periods when the gonad attains the maximum weight in relation to body weight, and so the more advances of maturity stages, it can be used as a maturity and breeding season index.

Descriptors: Micropogonias furnieri, reproduction (biology), condition factor, spawning seasons. Descritores: Micropogonias furnieri, reprodução biolögica, fator de condição, período de desova.

\section{Introdução}

0 fator de condição (K) em peixes varia de acordo com influências de fatores fisiológicos e ambientais, sendo conhecidas suas flutuações durante um ciclo sexual (Clark, 1934; Angelescu et al., 1958; Vazzoler \& Vazzoler, 1965), particularmente em exemplares adultos

(Bagenal, 1957).

De modo geral, o valor de $\mathrm{K}$ tem sido estimado como a razão entre o peso total do indivíduo e o cubo do comprimento total (Le Cren, 1951).

No presente trabalho, analisam-se variações do fator de condição, estimado como a constante a de relaçoes peso/comprimento, em função do desenvolvimento gonodal de adultos de corvina, Micropogonias furnieri (Desmarest, 1823), - qual foi avaliado por observação de características macro e microscópicas dos ovários. Visa-se a obter um índice para determinação das épocas de desova da espécie.

(*) Ex-bolsista da OEA e CNPq.

(**) Atualmente no Instituto Nacional de Pesquisas da Amazônia - INPA.

Publ. n. 583 do Inst. oceanogr. da Usp.

\section{Material e métodos}

Durante o período de fevereiro de 1979 a janeiro de 1980 e em abril de 1980 , foram coletadas 102 fêmeas adultas em Ubatuba, litoral norte do Estado de São Paulo, dentro da área ocupada pela população I da espécie (Vazzoler, 1971). Dessas fêmeas, anotaram-se dados sobre comprimento total (mm), peso total e dos ovārios (g) e estádio de maturação sexual, segundo critérios descritos por Vazzoler (1981).

Os ovārios de todas as fêmeas foram fixados em formol 10\%, sendo preparadas 1âminas histólogicas para controle microscópico dos estádios de maturação.

Associando-se às características macro e microscópicas, os ovärios das 102 fêmeas foram classificados em quatro estádios:

$$
\begin{aligned}
& \mathrm{R}=\text { repouso } \\
& \mathrm{B}=\text { em maturação } \\
& \mathrm{C}=\text { maturo } \\
& \mathrm{D}=\text { esvaziado }
\end{aligned}
$$

Foram calculadas as freqüências relativas mensais de ocorrência de cada es- 
tādio durante o período estudado (Fig. 1). Os dados de abril de 1980 foram considerados com representativos de abril de 1979, no qual não foi possível coletar fêmeas.

Foi estimada a relação entre peso total e comprimento total, através do ajuste da expressão $\mathrm{W}_{\mathrm{t}}=\mathrm{a} \mathrm{L}_{\mathrm{t}}^{\mathrm{b}}$ aos dados de peso e comprimento totais obtidos das fêmeas, apōs transformação logarítmica e aplicando o método dos mínimos quadrados, visando-se a obter o valor de " $b$ " para substituir o valor de 3 (cubo) na estimativa de $\mathrm{K}$ e $\mathrm{K}^{\prime}$.

0 valor individual do fator de condição foi calculado, considerando-se as seguintes expressões:

$$
K=\frac{\mathrm{W}_{\mathrm{t}}}{\mathrm{L}_{\mathrm{t}}^{\mathrm{b}}} \cdot 10^{5} \quad \mathrm{e}
$$
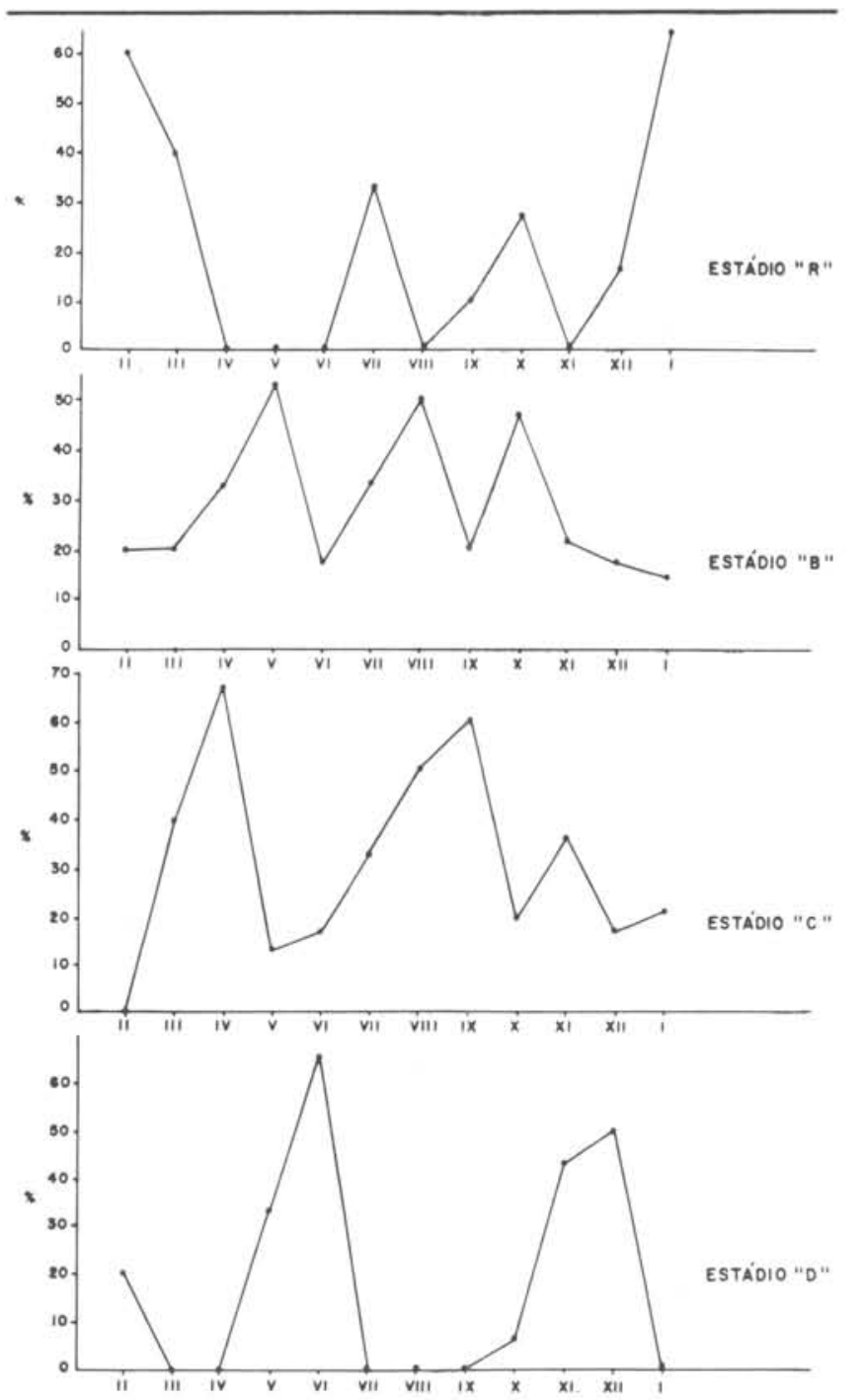

Fig. 1. Representação gräfica da frequência de ocorrência de estádios de maturidade, no período estudado.

$$
\begin{aligned}
& \mathrm{K}^{\prime}=\frac{\mathrm{W}_{\mathrm{t}}-\mathrm{W}_{\mathrm{g}}}{\mathrm{L}_{\mathrm{t}}^{\mathrm{b}}} \cdot 10^{5} \text {, onde: } \\
& \mathrm{K}=\text { fator de condição considerando-se } \\
& \mathrm{W}_{\mathrm{t}}=\text { peso total }(\mathrm{g}) \\
& \mathrm{W}_{\mathrm{g}}=\text { peso das gônadas }(\mathrm{g}) \\
& \mathrm{L}_{\mathrm{t}}=\text { comprimento total (mm) } \\
& \mathrm{b}=\text { estimador do coeficiente angular } \\
& \text { da regressão da relação } \log \\
& \mathrm{W}_{\mathrm{t}} / \log \mathrm{L}_{\mathrm{t}} \text {. }
\end{aligned}
$$

A seguir, calculou-se as médias mensais de $\mathrm{K}$ e $\mathrm{K}^{\prime}$ (Fig. 2), bem como a variação média de ambos ( $\overline{\Delta K})$ (Fig. 3), sendo:

$$
\overline{\Delta \mathrm{K}}=\overline{\mathrm{K}}-\overline{\mathrm{K}^{\top}}
$$

Os resultados foram analisados comparativamente (Fig. 4).

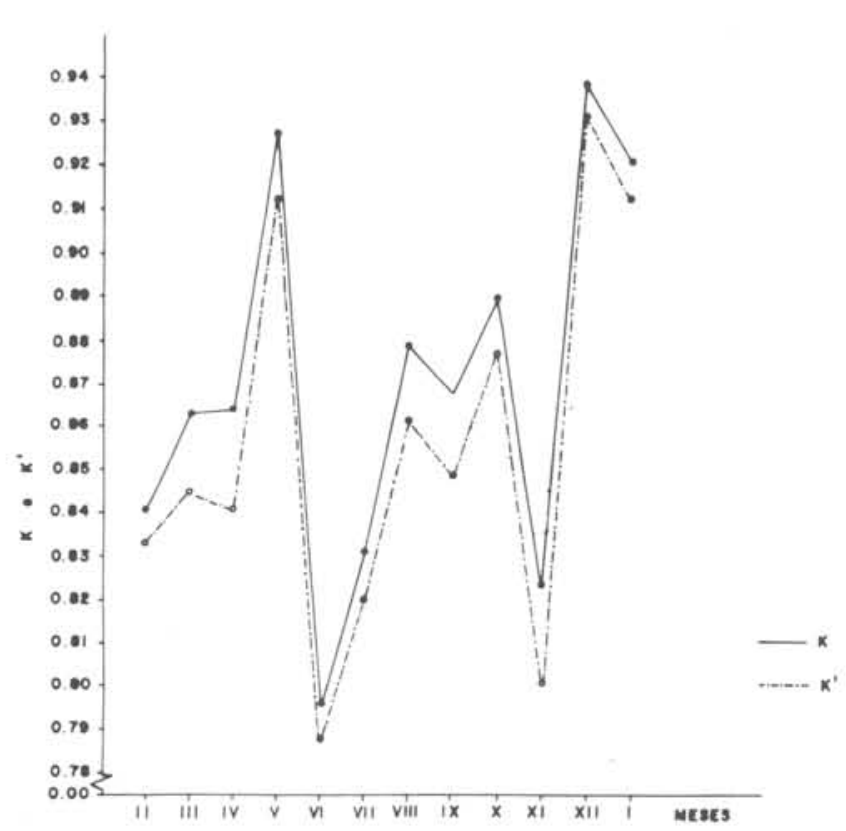

Fig. 2. Variação mensal dos valores médios do Fator de Condição Geral (K) e do Fator de Condição do Corpo $\left(K^{\prime}\right)$. 


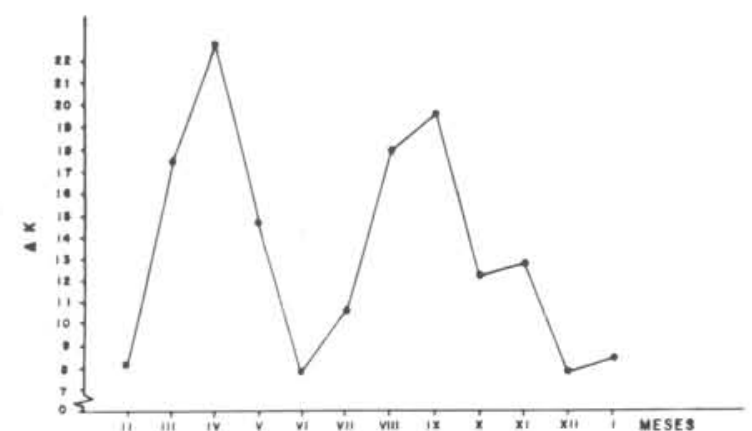

Fig. 3. Variação mensal da diferença dos dois Fatores de Condição ( $\mathrm{K}$ e $\mathrm{K}^{\prime}$ ).

\begin{tabular}{|c|c|c|c|c|c|c|c|c|c|c|c|c|}
\hline \multirow{2}{*}{$\begin{array}{l}\text { MESES } \\
\text { RESUEST } \\
\text { TADOS }\end{array}$} & $\begin{array}{l}\text { ABA } \\
\text { iv }\end{array}$ & MAI & $\begin{array}{l}\text { JUN } \\
\text { Vi }\end{array}$ & $\begin{array}{l}\text { Jut } \\
\text { VII }\end{array}$ & $\begin{array}{l}\text { AGO } \\
\text { Viii }\end{array}$ & $\begin{array}{c}\text { SE } 1 \\
\text { ix } \\
\end{array}$ & $\begin{array}{c}\text { OUT } \\
x\end{array}$ & $\begin{array}{c}\text { NOV } \\
\times 1\end{array}$ & 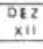 & $\begin{array}{c}\text { JAN } \\
1\end{array}$ & $\begin{array}{c}F E V \\
11\end{array}$ & $\begin{array}{l}\text { MAA } \\
\text { III }\end{array}$ \\
\hline & \multicolumn{3}{|c|}{ OUTONO } & \multicolumn{3}{|c|}{ INVERNO } & \multicolumn{3}{|c|}{ - primavera } & \multicolumn{3}{|c|}{ VEAAO } \\
\hline$\theta$ & & $\circ$ & & & 。 & & 0 & & & & & \\
\hline$c:$ & $a$ & & & & 9 & 9 & & $\alpha$ & & $a$ & & \\
\hline 0. & & & 0 & & $\backslash$ & $V$ & & $\sigma$ & 0 & & 0 & \\
\hline 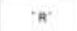 & & & & ० & & & 0 & & & 0 & 0 & \\
\hline ₹ & & 0 & & & $\sigma^{\prime}$ & & 0 & & 0 & & & \\
\hline$\kappa^{*}$ & & 。 & & & $\sigma^{-}$ & & 0 & & $\circ$ & & & \\
\hline$\Delta x$ & 0 & & & & 0 & 0 & & 0 & & & 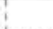 & \\
\hline DEsova & & & & & & IIS & & & & & & \\
\hline
\end{tabular}

Fig. 4. Quadro comparativo da ocorrência dos valores modais dos quatroestädios de maturidade, do fator de condição $\left(K\right.$ e $\left.K^{\prime}\right)$ e do $\Delta K$, com inferếncia sobre a época de desova de M. furnieri.

\section{Resultados}

Quanto às características macroe microscópicas dos ovārios e freqüência temporal (Fig. 1) dos quatro estādios de maturidade, observou-se que:

a) Ovários em repouso (estádio $R$ ): representam um período estacionário dos ovärios; estes apresentam-se translúcidos e ocupam menos da metade da cavidade abdominal. Os cortes desses ovārios mostram lamelas ovígeras apenas com células germinativas jovens dispostas ordenadamente. Ovārios neste estádio ocorrem em janeiro, fevereiro, março, julho, setembro, outubro e dezembro, com valores modais em janeiro-fevereiro, julho e outubro;

b) Ovărios em maturação (estádio B): iniciam seu desenvolvimento, apresentando-se amarelos e de aspecto granular devido aos ovócitos opacos, em maturação; ocupam cerca de metade da cavidade abdominal. Nos cortes histológicos, os ovócitos mais avançados apresentam gotas de gordura e vesículas de vitelo em seu ooplasma. Ovārios neste estādio ocorrem o ano todo, com modais em maio, agosto e outubro;

c) Ovários maduros (estädio C): são identificados por sua coloração amareloalaranjada e por seu tamanho; ocupam, praticamente, toda a cavidade abdominal. Os ovócitos são grandes e fluem pelo poro genital quando uma leve pressão é exercida no ventre da fêmea. Os cortes destes ovários mostram ovócitos maduros, com seu ooplasma repleto de vesículas de vitelo. As lamelas ovígeras apresentamse distendidas, devido à pressão dos ovócitos. Ovários neste estādio ocorrem o ano todo, com exceção de fevereiro, sendo os valores modais observados em abril, agosto-setembro e novembro, e

d) Ovários esvaziados (estádio D): apresentam coloração amarelo-avermel.hada, mostrando alto grau de vascularização e hemorragias, ocupando cerca de metade da cavidade abdominal. Manchas escuras de ovócitos em degeneração são observadas na superfície destes ovärios. Nos cortes, observam-se corpos foliculares (folículos esvaziados) que restaram após a desova; entre as lamelas ovígeras, vārios ovōcitos jovens estão prontos para reiniciar o ciclo. Numerosos capilares e vasos sanguíneos são vistos próximos às células em degeneração. Ovārios neste estádio ocorrem em fevereiro, maio, junho, outubro, novembro e dezembro, sendo que as modas verificam-se em fevereiro, junho e novembro-dezembro.

Ocorrem, para todos os estádios, três modas com continuidade temporal (Fig; 1). Considerando-se os estádios C, D, e $R$, temos que: no outono - início do inverno, ocorre uma moda de $\mathrm{C}$ em abril, seguida de uma de $D$ em junho e outra de $R$ em julho; no inverno - início da primavera, há uma moda de $\mathrm{C}$ em agosto-setembro e uma de R em outubro; na primavera - início do verão, verifica-se uma moda de $C$ entre novembro-janeiro, outra de $D$ entre novembro- fevereiro e uma terceira de $\mathrm{R}$ em janeiro-fevereiro.

Os valores estimados para os parâmetros da relação peso/comprimento totais para as fêmeas adultas foram: 0,8511 x $10^{-5}$ para a constante a e 3,03 para 0 coeficiente $b$, sendo 0,96 o valor do coeficiente de correlação linear de Pearson ( $r$ ).

O fator de condição mostrou flutuações acentuadas durante o ano (Fig. 2), 
sendo que os valores médios mensais variaram de $0,7957 \times 10^{-5}$ a $0,9386 \times 10^{-5}$ para $K$, de $0,7879 \times 10^{-5}$ a $0,9308 \times 10^{-5}$ para $K^{\prime}$ e de $7,8 \times 10^{-8}$ a $22,8 \times 10^{-8}$ para $\Delta K$.

Tanto $\mathrm{K}$ como $\mathrm{K}^{\prime}$ mostraram três picos, sendo um em maio, outro em agosto-outubro e um terceiro em dezembro (Fig. 2). Os valores mais baixos ocorreram nos meses de junho, novembro e fevereiro, períodos que coincidem com os de mais alta freqüência de ovārios em estádio D, esvaziados.

Apesar de $\mathrm{K}$ e $\mathrm{K}^{\prime}$ apresentarem a mesma tendência em sua variação anual, não hā um paralelismo absoluto nos mesmos, havendo períodos em que os valores de $\Delta K$ tornam-se mais elevados (Fig. 3). Observam-se três picos de $\Delta K$, nos meses de maio, agosto-setembro e novembro, coincidindo com os períodos de máxima ocorrência de ovários maturos, em estādio C.

Deste modo, pode-se dizer que $\Delta \mathrm{K}$ estima a "condição da gônada", estabelecendo os períodos em que os ovärios apresentam-se com peso máximo em relação ao peso do indivíduo, tendo atingido maturidade máxima.

$\mathrm{Na}$ Figura 4, observam-se, conjuntamente, os resultados obtidos, comparando os valores modais do $K, K^{\prime}$ e $\Delta K$ aos de ocorrência dos estádios de maturidade, fundamentalmente de C e D. Distingue-se, assim, claramente, a coincidência temporal destes valores, delimitando três picos de desova, nos períodos localizados entre os valores modais dos estádios $\mathrm{C}$ e D.

De acordo com estas evidências, conclui-se que Micropogonias furnieri desovou três vezes no período e ärea estudados, ocorrendo uma desova no outono, entre abril e junho, uma segunda no inverno, entre agosto e setembro, e ima terceira no fim da primavera-verão, entre novembro e fevereiro.

\section{Discussão}

As modalidades de reproduçãoe, em particular, o rítmo de desova representam adaptações do organismo ao meio ambiente.

Brewer (1978) provocou, experimentalmente, que Engraulis mordax, quando submetida a uma temperatura entre $13^{\circ}$ e $18^{\circ} \mathrm{C}$ e com suprimento alimentar adequado, pode reproduzir-se o ano todo, sugerindo que esses dois fatores determinam a sazonalidade do ciclo sexual em condições naturais.
Após a primeira maturidade sexual, a maior parte do alimento ingerido è utilizada para o desenvolvimento das gonodas e as reservas de gordura para o inverno (Nikolsky, 1963). Importantes modificações no metabolismo, particularmente dos lípides e proteínas, ocorrem ao longo da maturação sexual (Fontaine \& Olivereou, 1962).

Diferenças no fator de condição têm sido interpretados como medidas de vārios eventos biológicos, tais como reserva de gordura, adequação ao meio ambiente e desenvolvimento gonadal (Le Cren, 1951). Freqüentemente, o fator de condição é correlacionado com ciclos reprodutivos, sendo suas variações explicadas pela intensa atividade das gonadas de peixes maduros (Hickling, 1936; Bagena1, 1957; Vazzoler \& Vazzoler, 1965).

Em alguns casos, o valor de $\mathrm{K}$ resulta mínimo, quando a freqüência de indivíduos maduros é elevada (Vazzoler \& Vazzoler, op. (it.) ou quando a gônada estā em seu máximo de maturidade (Conand, 1977), representando, nestes casos, mais um indicador de períodos de alimentação e jejum, que podem estar 1igados ao ciclo reprodutivo. Em outros casos, não se verifica qualquer relação entre a condição e o ciclo gonadal (Blackburn, 1960).

0 fator de condição, em função de sua variação, constitui-se em elemento quantitativo importante para a avaliação da relação entre a forma do corpo e seu peso relativo. 0 desenvolvimento das gonadas femininas ocorre em ritmo mais intenso que o incremento, em comprimento ou peso do peixe, tendo, como conseqüência, uma mudança mais acentuada na forma do corpo das fêmeas ao longo do ciclo sexual. Isto se manifesta com um aumento do peso total e, consequentemente, de $\mathrm{K}$, de carāter temporārio, quando a gônada atinge seu mäximo tamanho, antes da desova (Angelescu et al., 1958).

Vazzoler (1971) constatou que a população de M. furnieri (população I) que ocorre na área entre $23^{\circ}$ e $29^{\circ} \mathrm{S}$ desova no inverno e na primavera; deve-se ressaltar, entretanto, que nesse estudo não foram obtidas amostras nos meses de abril e maio, o que explicaria a não detecção da desova de outono. A mesma autora delimita que a população desta espécie, que habita mais ao sul entre os $29^{\circ}$ e $33^{\circ} \mathrm{S}$ (população II), desova durante a primavera e verão. 
Daneri (1957) e Haimovici (1977), estudando a mesma espécie (M. opercularis = M. furnieri) em Mar del Plata (Argentina), determinam um ciclo anual com desova apenas no verão. Lowe-McConnel1 (1966) sugere que a reprodução de $M$. furnieri na costa da Guiana não tem caräter sazonal, sendo contínua, vista a ocorrência tanto de fêmeas maduras como a de alevinos durante todo o ano.

Considerando as diferenças na periodicidade do ciclo reprodutivo ao longo da ārea de ocorrência de M. furnieri, sugere-se que elas estejam associadas às características oceanogrä́ficas de cada uma das regiões citadas. Segundo Lopez (1963), na costa argentina existe uma faixa costeira, cuja maior largura ocorre na frente da Província de Buenos Aires (40-45 braças no verão), correspondendo a uma massa de água de origem subtropical, que, bordeando a costa, pode alcançar, no verão, latitudes mais altas que Cabo Blanco, ao Sul dos $46^{\circ} \mathrm{S}$. Estas äguas, conhecidas como Āguas Velhas da Plataforma (Hart, 1946), são substituídas parcialmente no inverno pela Corrente das Malvinas, que se aproxima da costa nesta época trazendo äguas muito mais frias.

As águas da plataforma sudeste do Brasil são constituídas principalmente por águas subtropicais, misturadas com ăguas tropicais e costeiras (Emílsson, 1961). Nesta ārea, distinguem-se três regiões: 1) de Cabo Frio até São Sebastião, com águas mais frias e produtivas, devido à freqüente mas não periódica ocorrência de ressurgências, fundamentalmente no final da primavera, verão e início do outono; 2) do norte de Santos até - sul do Estado de Santa Catarina, com características mais estāveis e flutuações apenas devidas às mudanças climáticas, e 3) litoral do Rio Grande do Sul: zona de transição, com grandes flutuações sazonais de temperatura e salinidade devido às mudanças na posição da Convergência Subtropical (Kawakami de Resende, 1979). Cabe lembrar que a população I de $M$. furnieri ocupa as regiões delimitadas nos itens 1 e 2 , sendo que as coletas para este estudo foram efetuadas apenas na região delimitada em 1 .

As características ambientais do habitat marinho do 1itoral da Guiana apresentam condições estáveis, com äguas de origem tropical durante todo o ano, provindas do Giro Norte da Corrente Sul-
Equatorial. As temperaturas superficiais oscilam entre $24^{\circ}$ e $29^{\circ}$, com médias de $25^{\circ}$ e $27^{\circ}$ para o inverno e verão, respectivamente (U.S. Naval Oceanographic Office, 1967).

Assim, parece evidente que os fatores ambientais, em especial a temperatura, teriam efeito sobre a fisiologia do ciclo, delimitando, em cada região, qual o período de produção de ovos e de desova. $\mathrm{E}$ as massas de água determinariam, em cada região, a sazonalidade e a duração do ciclo de produção de plâncton, que servirá de alimento às larvas após a eclosão. Nas áreas em que esta produção ocorre em épocas delimitadas, a desova deverá ocorrer de maneira tal que a eclosão coincida temporalmente com o período de maior disponibilidade de alimento. De uma forma ou de outra, mecanismos adaptativos permitem aos peixes desovarem em um tempo tal que assegure a suas larvas uma boa alimentação. Caso a produção de plâncton seja contínua ao longo do ano, não hä necessidade de tal mecanismo (Cushing, 1977; Nikolsky, 1963).

Almeida-Perez (1972) estabeleceu que a "corvina" Cynoscion maracaibaensis, da Venezuela, desova o ano todo, com um período principal de janeiro a maio e períodos secundārios em junho e setembro.

Bagenal (1957) verificou que o fator de condição de Hipoglossoides

platessoides é mais baixo nos peixes imaturos que nos maduros; durante os meses de desova, à medida em que os indivíduos vão desovando, perdem condições rapidamente, sendo que os $K$ das fêmeas com ovārios esvaziados correspondem aos mais baixos valores encontrados.

Juras (1979) constatou, para Macrodon ancylodon, que os picos nos valores do fator de condição ocorriam nos períodos imediatamente anteriores à desova.

Estes resultados, quanto à variação dos valores de $\mathrm{K}$ e $\mathrm{K}^{\prime}$ durante o ciclo reprodutivo de $M$. furnieri, mostram que ocorre superposição de seus valores māximos (modas) com os picos de ocorrência do estádio C (pré-desova) e, de seus mínimos, com os máximos de ocorrência do estádio D (pós-desova); em concordância com o constatado pelos autores anteriormente discutidos, essas variações seriam explicadas pelo acúmulo e posterior gasto de reservas que ocorrem durante a maturação e desova. Mostram também que as maiores diferenças entre o $\mathrm{K}$ e o $\mathrm{K}^{\prime}$ verificam-se nas épocas em que a gônada 
Bolm Inst. oceanogr., S Paulo, 32(1), 1983

está no máximo de atividade, fato que justifica o uso de $\Delta \mathrm{K}$ como um indice indireto de maturidade e dos períodos de desova de M. furnieri.

\section{Resumo}

A anālise temporal da freqüência de estādios de maturação de 102 fêmeas adultas coletadas entre fevereiro de 1979 e janeiro de 1980 na região de Ubatuba, litoral norte do Estado de São Paulo, mostra que M. furnieri, nessa ārea, apresenta três desovas durante o ano: uma no outono (abril-junho), outra no inverno (agosto-setembro) e uma tereeira na primavera-verão (novembro-feverelro). Essa sugestão é reforçada pelas flutuações de K (fator de condição considerando-se o peso das gônadas) e K' (sem considerar o peso das gônadas), durante um ciclo reprodutivo. Os valores mäximos de $\mathrm{K}$ e $\mathrm{K}^{\prime}$ coincidem com os picos de ocorrência de indivíduos com gônadas maturas (C) e, os mínimos, com os de indivíduos com gônadas esvaziadas (D). A diferença entre $K$ e $K^{\prime}(\Delta R)$ estima a "condição da gônada" apresentando seus máximos nos períodos de mais intensa atividade dos ovários, contituindo-se, assim, o valor de $\Delta K$ em um indice indireto de maturidade e dos períodos de desova de M. furnieri.

\section{Agradecimentos}

Agradecemos ao Instituto Oceanogräfico da Universidade de São Paulo, ao funcionários da Base Norte do Instituto, aos docentes e funcionārios do Instituto de Ciências Biomédicas (Departamento de Histologia) da Universidade de São Paulo, à Dra. Carmen Lúcia Del B, RossiWongtschowski, à Srta. Lucy Teixeira e a todos os que, de alguma forma, colaboraram para a realizaçãa deste traba1ho.

\section{Referências bibliográficas}

ALME IDA-PEREZ, P. 1972. Estudio preliminar de la histocitologia de los ovários de la corvina Cynoscion maracaibaensis, Schultz, del lago de Maracaibo (Venezuela). (Perciformés, Sciaenidae). Mem Soc. cient. natur. "La Sal1e", 32 (91):20-46.

ANGELESCU, V.; GNERI, F, S. \& NANI, A. 1958. La merluza del mar argentino (biologia y taxonomia) Serv. Hidrog. nav., B Aires, (H 1004):1-224.
BAGENAL, T, B. 1957. The breeding and fecundity of the long rough dab Hippoglossoides platessa (Fabr.) and the associated cycle in condition. J. mar. biol. Ass. U. K., 36:339-375.

BLACKBURN, M. 1960. A study of condition (weight for length) of Australian barracouta Thyrsites atun (Euphrasen). Austr. J. Mar. Freshw. Res., 11 (1):14-41.

B.REWER, G. D. 1978. Reproduction and spawning of the northern anchovy Engraulis mordax in San Pedro Bay, C.alifornia. Calif. Fish Game, 64 (3) : $175-184$.

CLARK, F. N. 1934. Maturity of California sardine determined by ova diameter measurementș: Fish. Bu11. C1.if., 42:1=49.

CONAND, c. 1:977. Contribution à 1 'etude du cycle sexual et de la fecundité de la sardinella ronde, Sardinella aurita: pêche sardiniere da Karoi se en 1975, et premier semestre 1976. Cah. O.R.S.T.O.M., sér. Océanogr., 15(14):301-312.

CUSHING, D. H. 1977. Science and the fisheries. Studies in biology No 85. London, Edward Arnold (publishers) Ltd., 60 p.

DANERI, C. A. 1957. Contribucíón a.1 conocimiento del ciclo sexual de la corvina blanca Micropogon opercularis. (Quoy y Gainnard). Boln. Mus. argent. Cient. Nat. Bernardino Rivadavia, 14:1-.24.

EMILSSON, I. 1961. The shelf and coastal vaters off southern Brazil. Boln Inst. oceanogr., S Paulo, $11(2)$ : 101-112.

FONTAINE, M. \& OLIVEREAU, M. 1962. Nutrition et sexualité chez les poísson. Am. Nutr. Aliment., $16(6): 125-152$.

HAIMOVICI, M. 1977. Idade, crescimento e aspectos gerais da biologia da corvina Micropogon opercularis (Quoy e Gaimard, 1824) (Pisces, Scianidae). At1ântica, R Griande, 2(1):21-49. 
HART, T. J. 1946. Report on trawling surveys on the Patagonian continental shelf. Discovery Rep., 23:223-408.

HICKLING, C. F. 1936. Seasonal changes in the ovary of the inmature hake, Merlucius merlucius L. J. mar. biol. Ass. U.K., 20(2):443-461.

JURAS, I. da A. G. M. 1979. Estudo sobre o crescimento de Macrodon ancylodon (Bloch \& Schneiter: 1801) capturada nas costas do Rio Grande do Sul (Latitude $29^{\circ} \mathrm{S}$ a $32^{\circ} \mathrm{S}$ ). Dissertação de mestrado. Universidade de São Paulo, Instituto Oceanogräfico, $182 \mathrm{p}$.

KAWAKAMI de RESENDE, E. 1979. Estudo da distribuição, estrutura, biologia e bionomia de Syacium papillosum (Linnaeus, 1758), na plataforma continental brasileira entre Cabo Frio $\left(23^{\circ} \mathrm{S}\right)$ e Torres $\left(29^{\circ} 21^{\prime} \mathrm{S}\right)$. Tese de doutorado. Universidade de São Pau1n, Instituto Oceanogräfico, 198p.

LE CREN, E. D. 19.51. The lengthweight relationship and seasonal cycle in gonad weight and condition in the perch (Perca fluviatis). J. Anim. Eco1., 20(2):201-219.

LOPEZ, R. B. 1963. Peces marinos de 1a República Argentina. Cons Fed. Inv., Eval. Recur. nat. Arg., 7(3): 105-116.

LOWE-McCONNELL, R. H. 1966. The Sciaenidae fishes of British Guiana. Bul1. mar. Sci., 16(1):20-57.
NIKOLSKY, G. V. 1963. The ecology of fishes. London, Academic Press, : 315 . 328 .

U.S. NAVAL OCEANOGRAPHIC OFFICE. 1967. Oceanographic atlas of the North Atlantic Ocean. Section II- Physical properties. Publication 700,300 p.

VAZZOLER, A. E. A. de M. 1971. Diversificação fisiológica e morfológica de Micropogon furnieri (Desmarest, 1822) ao sul de Cabo Frio, Brasil. Bolm Inst. oceanogr., S Paulo, 20(2): $1-70$.

\section{Manua1}

de métodos para estudos biológicos de populações de peixes. Reprodução e crescimento. Brasília, CNPq. Programa Nacional de Zoologia, 108p.

1965. Relation between condition factor and sexual development in Sardinella aurita (Cuv. \& Va1.). Anais Acad. bras. Ciênc., 37 (sup1.): 353-359.
(Recebido 18-maio-1982; ace i to 02-dez-1982) 